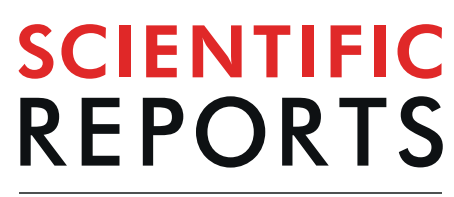

natureresearch

\title{
A 4-gene signature from histologically normal surgical margins predicts local recurrence in patients with oral carcinoma: clinical validation
}

\author{
Patricia P. Reis $\mathbb{1}^{1,12^{*}}$, Tomas Tokar ${ }^{2,12}$, Rashmi S. Goswami ${ }^{3}$, Yali Xuan ${ }^{4}$, Mahadeo Sukhai ${ }^{4}$, \\ Ana Laura Seneda ${ }^{1}$, Luis E. S. Móz ${ }^{5}$, Bayardo Perez-Ordonez ${ }^{6}$, Colleen Simpson ${ }^{4}$, \\ David Goldstein $\mathbb{1}^{4}$, Dale Brown ${ }^{4}$, Ralph Gilbert ${ }^{4}$, Patrick Gullane ${ }^{4}$, Jonathan Irish ${ }^{4}$, \\ Igor Jurisica ${ }^{2,7,8,9 *} \&$ Suzanne Kamel-Reid ${ }^{4,10,11^{*}}$
}

Prognostic biomarkers for recurrence of Oral Squamous Cell Carcinoma (OSCC) are urgently needed. We aimed to independently validate a 4-gene expression signature (MMP1, COL4A1, P4HA2, THBS2) predictive of OSCC recurrence risk. Gene expression was measured using Nanostring nCounter ${ }^{\circledR}$ in 245 histologically normal surgical resection margins from 62 patients. Association between risk scores for individual patients and recurrence was assessed by Kaplan-Meier analysis. Signature performance was quantified by concordance index $(\mathrm{Cl})$, hazard ratio $(\mathrm{HR})$ and the area under receiver operating characteristics (AUC). Risk scores for recurrence were significantly higher than recurrence-free patients $(p=9.58 \mathrm{e}-7$, Welch's t-test). A solid performance of the 4-gene signature was determined: $\mathrm{Cl}=0.64$, $\mathrm{HR}=3.38(p=1.4 E-4$; log-rank test $), \mathrm{AUC}=0.71$. We showed that three margins per patient are sufficient to preserve predictive performance $(\mathrm{Cl}=0.65 ; \mathrm{HR}=2.92 ; p=2.94 \mathrm{e}-3 ; \mathrm{AUC}=0.71)$. Association between the predicted risk scores and recurrence was assessed and showed $\mathrm{HR}=2.44(p=9.6 \mathrm{E}-3$; logrank test, $\mathrm{N}=62$ ). Signature performance analysis was repeated using an optimized threshold $\left(70^{\text {th }}\right.$ percentile of risks), resulting in $\mathrm{HR}=3.38(p=1.4 E-4$; log-rank test, $\mathrm{N}=62)$. The 4-gene signature was validated as predictive of recurrence risk in an independent cohort of patients with resected OSCC and histologically negative margins, and is potentially applicable for clinical decision making on adjuvant treatment and disease monitoring.

Oral Squamous Cell Carcinoma (OSCC) is the most common head and neck cancer, and the $8^{\text {th }}$ most frequent cancer $^{1}$, accounting for $>300,000$ new cases and $>145,000$ deaths every year, worldwide ${ }^{2}$. Conventional treatment for OSCC includes surgery, radiotherapy and chemotherapy. However, patients often exhibit poor response to treatment and high rates of recurrence, typically between $20-40 \%^{3}$, reducing the five-year survival to $50 \%{ }^{4}$.

\footnotetext{
${ }^{1}$ São Paulo State University, UNESP, Faculty of Medicine, Department of Surgery and Orthopedics, Botucatu, SP, Brazil. ${ }^{2}$ Krembil Research Institute, University Health Network, Toronto, ON, Canada. ${ }^{3}$ Department of Clinical Pathology, Sunnybrook Health Sciences Centre, Toronto, ON, Canada. ${ }^{4}$ Princess Margaret Cancer Centre, University Health Network, Toronto, ON, Canada. ${ }^{5}$ Brazilian Institute for Cancer Control, São Paulo, SP, Brazil. ${ }^{6}$ Department of Pathology, Toronto General Hospital, University Health Network, Toronto, ON, Canada. ${ }^{7}$ Departments of Medical Biophysics, University of Toronto, Toronto, ON, Canada. ${ }^{8}$ Department of Computer Science, University of Toronto, Toronto, ON, Canada. ${ }^{9}$ Institute of Neuroimmunology, Slovak Academy of Sciences, Bratislava, Slovakia. ${ }^{10} \mathrm{Clinical}$ Laboratory Genetics, Genome Diagnostics, University Health Network, Toronto, ON, Canada. ${ }^{11}$ Department of Laboratory Medicine and Pathobiology, The University of Toronto, Toronto, ON, Canada. ${ }^{12}$ These authors contributed equally: Patricia P. Reis and Tomas Tokar. *email: patricia.reis@unesp.br; juris@ai.utoronto.ca; suzanne.kamelreid@uhn.ca
} 


\begin{tabular}{|c|c|c|c|c|}
\hline & & \multicolumn{3}{|l|}{ Value } \\
\hline \multirow{2}{*}{\multicolumn{2}{|c|}{ Characteristics }} & $\begin{array}{l}\text { Current study } \\
\text { (independent validation) }\end{array}$ & \begin{tabular}{|l|}
$\begin{array}{l}\text { Reis et al. } 2011 \\
\text { (training) }\end{array}$ \\
\end{tabular} & \begin{tabular}{|l}
$\begin{array}{l}\text { Reis et al. } 2011 \\
\text { (validation) }\end{array}$ \\
\end{tabular} \\
\hline & & $\mathrm{N}=62$ & $\mathrm{~N}=\mathbf{2 4}$ & $\mathbf{N}=\mathbf{3 0}$ \\
\hline \multirow{2}{*}{ Sex: } & Female & 27 & 9 & 9 \\
\hline & Male & 35 & 15 & 21 \\
\hline \multirow{3}{*}{ Age [years]: } & Median & 64.5 & 58.5 & 67 \\
\hline & Mean & 62.7 & 59.4 & 65.2 \\
\hline & Range & $27-83$ & $37-83$ & $48-85$ \\
\hline \multirow{4}{*}{ Tumor stage: } & I & 11 & 1 & 4 \\
\hline & II & 17 & 7 & 9 \\
\hline & III & 15 & 3 & 7 \\
\hline & IV & 15 & 13 & 10 \\
\hline \multirow{3}{*}{ Tumor grade: } & Well & 17 & 0 & 0 \\
\hline & Moderate & 41 & 18 & 23 \\
\hline & Poor & 2 & 6 & 7 \\
\hline \multirow{2}{*}{ Recurrence: } & No & 28 & 15 & 23 \\
\hline & Yes & 34 & 9 & 7 \\
\hline \multirow{3}{*}{$\begin{array}{l}\text { Time to recurrence } \\
\text { [months]: }\end{array}$} & Median & 10.5 & 3.2 & 8 \\
\hline & Mean & 17.6 & 12.2 & 15.1 \\
\hline & Range & $2.9-86.3$ & $1.8-34$ & $2-36$ \\
\hline \multirow{3}{*}{ Follow-up [months]: } & Median & 63 & 13 & 21 \\
\hline & Mean & 75 & 24.8 & 26.7 \\
\hline & Range & 5-199 & $1.7-58.8$ & $1-81$ \\
\hline \multirow{3}{*}{ Smoking: } & Never & 23 & 8 & 5 \\
\hline & Former & 15 & 16 & 25 \\
\hline & \begin{tabular}{|l} 
Current \\
\end{tabular} & 23 & 0 & 0 \\
\hline \multirow{2}{*}{ Alcohol consumption: } & No & 20 & 7 & 9 \\
\hline & Yes & 40 & $\begin{array}{ll}17 \\
\end{array}$ & 21 \\
\hline
\end{tabular}

Table 1. Clinicopathological characteristics of patients in this study and in Reis et al. ${ }^{9}$.

One important factor associated with improved patient survival is complete surgical removal of the primary tumor ${ }^{3}$. Presence of epithelial dysplasia or tumor cells in the surgical resection margins is known to be associated with a higher risk of disease recurrence ${ }^{5}$. However, even with histologically normal surgical resection margins, a $10-30 \%$ recurrence rate is still observed ${ }^{6}$. Therefore, histologically normal margins may harbor molecular changes, leading to their malignant transformation and disease recurrence ${ }^{7,8}$. Previous studies identifying molecular changes in surgical margins in head and neck cancer, including OSCC, are outlined in Supplementary Table 1, with corresponding literature citations. Most of the previous work used a candidate gene or protein approach instead of global profiling, and were based on the analysis of distinct cancer sites in the head and neck, which have different biological and clinical behaviors. More importantly, there is a lack of comprehensive validation using independent samples.

In our previous work ${ }^{9}$, we identified a 4-gene signature (MMP1,COL4A1, P4HA2 and THBS2) predictive of OSCC local recurrence in a set of 96 margins from 24 patients, and validated our findings in an independent set of 136 histologically normal surgical margins from 30 OSCC patients. Here, we provide extended, independent, retrospective validation of this signature on a new, independent cohort of 245 histologically normal surgical margins from 62 patients with OSCC, using the NanoString nCounter assay ${ }^{10}$. This analysis confirmed that the 4-gene signature accurately predicts recurrence risk in patients with OSCC and histologically normal (negative) surgical resection margins.

\section{Results}

Patient characteristics. Out of 62 patients, 34 (55\%) had local recurrence, with median time to recurrence of 10.5 months after surgery (Table 1 ). There was a statistically significant association between recurrence and age $(\mathrm{CI}=0.57, \mathrm{HR}=2.35, \mathrm{p}=1.36 \mathrm{e}-2, \log$-rank test, $\mathrm{AUC}=0.62)$, but not gender, smoking status, alcohol consumption, tumor stage, grade, or tumor subsite (Table 2). Interestingly, no significant association between recurrence and age or other clinical characteristics was found in our previous study'.

Validation of the signature. For each of the margin samples we first calculated sample-specific risks, derived from signature gene expression values as obtained from individual samples (Supplementary Fig. 1). We compared the risks derived from the samples of patients with recurrence to those from patients without recurrence. We found that risks derived from recurrence-positive samples $(n=34 ; 55 \%)$ were significantly higher than the risk score from recurrence-negative samples ( $\mathrm{p}=9.58 \mathrm{e}-7$, Welch's t-test; Fig. 1 ). 


\begin{tabular}{|l|l|l|l|l|}
\hline Characteristics & HR & $\boldsymbol{p}$-value & $\boldsymbol{N}$ & $\boldsymbol{n}$ \\
\hline Age & 2.35 & 0.0136 & 62 & 2 \\
\hline Sex & 0.86 & 0.6517 & 62 & 2 \\
\hline Tumor stage & 1.26 & 0.7880 & 58 & 4 \\
\hline Tumor grade & 2.29 & 0.1561 & 60 & 3 \\
\hline Smoking status & 1.36 & 0.0732 & 61 & 3 \\
\hline Alcohol consumption & 0.61 & 0.1632 & 60 & 2 \\
\hline Tumor subsite & 1.22 & 0.5704 & 62 & 2 \\
\hline
\end{tabular}

Table 2. Prognostic ability of clinical characteristics. $N$ denotes number of patients used and $n$ indicates number of groups, patients were stratified into, to assess the given characteristics. In case of age assessment patients were stratified using median age (64.5) as a threshold.

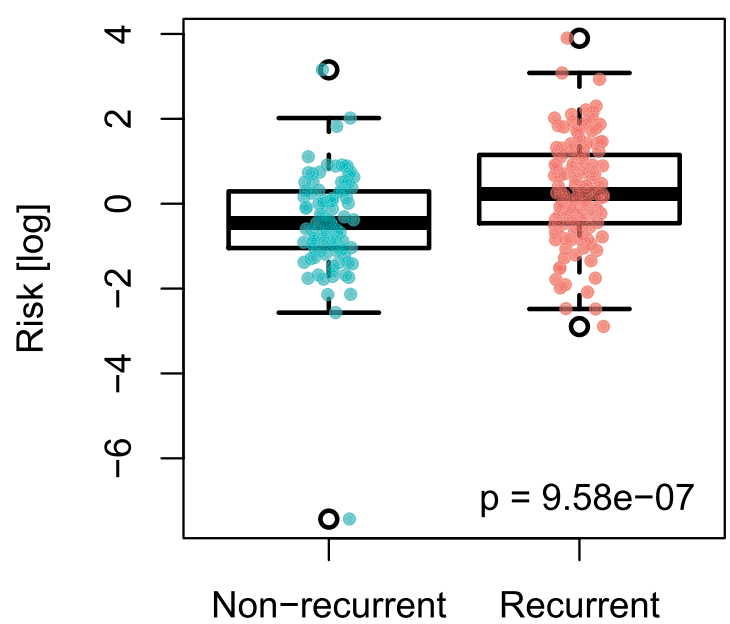

Figure 1. Boxplot depicting distribution of risks derived from margins samples of patients without $(\mathrm{n}=28$; $45 \%)$ and with locally recurrent tumors $(n=34 ; 55 \%)$.

For each patient, we then predicted the risk of local recurrence using the maximum gene expression value across all margins. The 4-gene signature was first evaluated by concordance index (CI), between predicted risk and recurrence-free survival time. The resulting value $(\mathrm{CI}=0.64)$ indicated a solid predictive performance of the signature. The area under the receiver operating characteristics curve (AUC) was calculated, resulting in $\mathrm{AUC}=0.71$, further highlighting the predictive performance of the signature (Fig. 2). Calculating Spearman rank correlation between predicted risks and age at diagnosis showed $\rho=-0.047(\mathrm{p}=0.71$, randomization test), excluding possible confounding effect of age. Patients were then stratified into two groups using median risk as a threshold. Association of the predicted risk and recurrence was assessed by calculating hazard ratio (HR). The resulting values: $\mathrm{HR}=2.44$ ( $\mathrm{p}=9.6 \mathrm{E}-3$; log-rank test, $\mathrm{N}=62$; Fig. $3 \mathrm{a}$ ) validated our published results ${ }^{9}$. This was then repeated using an optimized threshold $\left(70^{\text {th }}\right.$ percentile of risks), resulting in $\mathrm{HR}=3.38(\mathrm{p}=1.4 \mathrm{E}-4$; log-rank test, $\mathrm{N}=62$; Fig. 3b).

To assess how the reduced number of margin samples affects the 4-gene signature performance, we predicted the recurrence risk for each patient using gene expression from only one, randomly selected margin sample. CI, AUC and HR (using $70^{\text {th }}$ percentile of risks as a threshold) with their respective p-values, were subsequently determined to evaluate the predictive performance. This was repeated 10,000 times, resulting in median values of $\mathrm{HR}=1.63$ ( $\mathrm{p}$-value $=0.16$, log-rank test, $\mathrm{N}=62$ ), $\mathrm{CI}=0.59, \mathrm{AUC}=0.63$ (Fig. 4). Resulting values demonstrate substantial decline of the signature performance. This further corroborates our previous results ${ }^{9}$, leading to the conclusion that expression changes of this 4-gene signature are spread heterogeneously across the margins, and the use of only one or a reduced number of margins for molecular analysis decreases the probability of detecting gene expression changes associated with recurrence.

To further investigate how many margins are needed to provide robust recurrence prediction, the same Monte-Carlo analysis was applied herein, using maximum expression of genes, as calculated across two and three randomly selected margins per patient. Patients with insufficient number of margins samples were omitted. Using two different margins samples per patient resulted in median values of: $\mathrm{HR}=2.49$ ( $\mathrm{p}$-value $=8.1 \mathrm{e}-3, \log$-rank test, $\mathrm{N}=57$ ), $\mathrm{CI}=0.61, \mathrm{AUC}=0.68$ (Fig. 4). Using three different margin samples per patient results in median values of: $\mathrm{HR}=2.92$ ( $\mathrm{p}$-value $=2.94 \mathrm{e}-3$, log-rank test, $\mathrm{N}=47$ ), $\mathrm{CI}=0.67, \mathrm{AUC}=0.73$ (Fig. 4), comparable to the values observed when using a full set of samples across the whole cohort of patients.

Functional assessment of the signature genes. To provide functional analysis of the signature genes we further performed a comprehensive pathway enrichment analysis using validated head and neck tissue-specific 


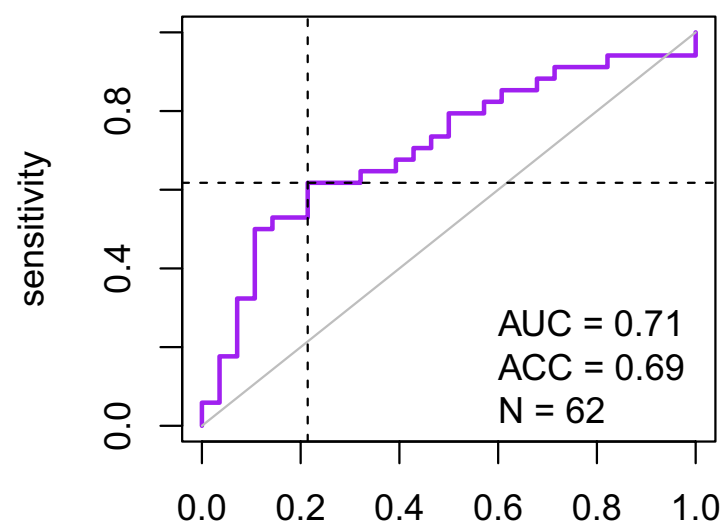

1- specificity

Figure 2. Receiver operating characteristics depicting patient classification performance of the signature. Horizontal and vertical lines indicate sensitivity and false positive rate ( 1 - specificity) achieved when using median risk as the threshold. Associated statistics, including area under the curve and accuracy, are listed in the bottom right corner.

A

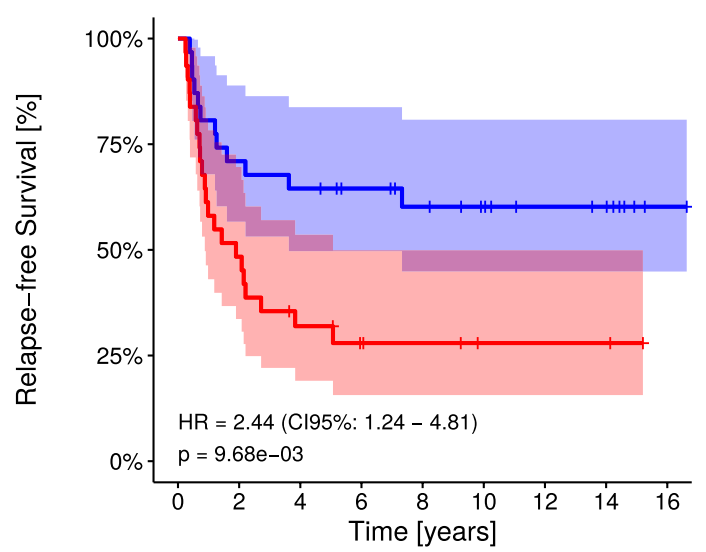

Number at risk

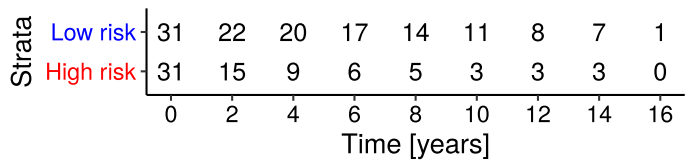

B $\quad$ Strata + Low risk + High risk

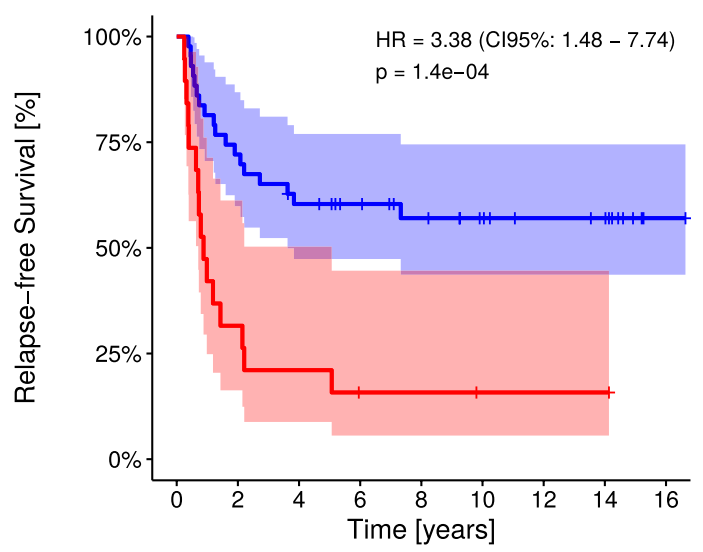

Number at risk

Figure 3. Kaplan-Meier plot depicting survival of the patients with low risk (LR) and high risk (HR) as estimated by applying the signature, stratifying patients using the median of the obtained risks (A) and optimized threshold, equal to $70^{\text {th }}$ percentile of the obtained risks. (B) Colour-shaded areas depict $95 \%$ confidence intervals of the survival curves. Obtained statistics are listed in the bottom left corner.

PPI partners. We identified 43 significantly enriched pathways (FDR $<0.01$; Supplementary Fig. 2). The most enriched pathways include organization of extracellular matrix, cell surface interactions of Beta1 and Beta3 integrins, collagen synthesis, focal adhesion, PI3K-Akt signalling, and miRNA targets in extracellular matrix and membrane receptors.

\section{Discussion and Conclusions}

Prognostic models require independent validation studies before can be considered as clinically useful ${ }^{11}$. Therefore, there is a need to demonstrate an ability to distinguish patients with favorable from poor outcome across multiple independent cohorts, and with an alternative analysis method. In order to satisfy these requirements, we performed an extended validation of the previously developed 4-gene prognostic signature, using an independent cohort of patients as well as an alternative, probe-based assay (Nanostring nCounter ${ }^{\circledR}$ ) for obtaining gene expression data. The limitation of this study is the lack of participants from geographically distinct populations. 

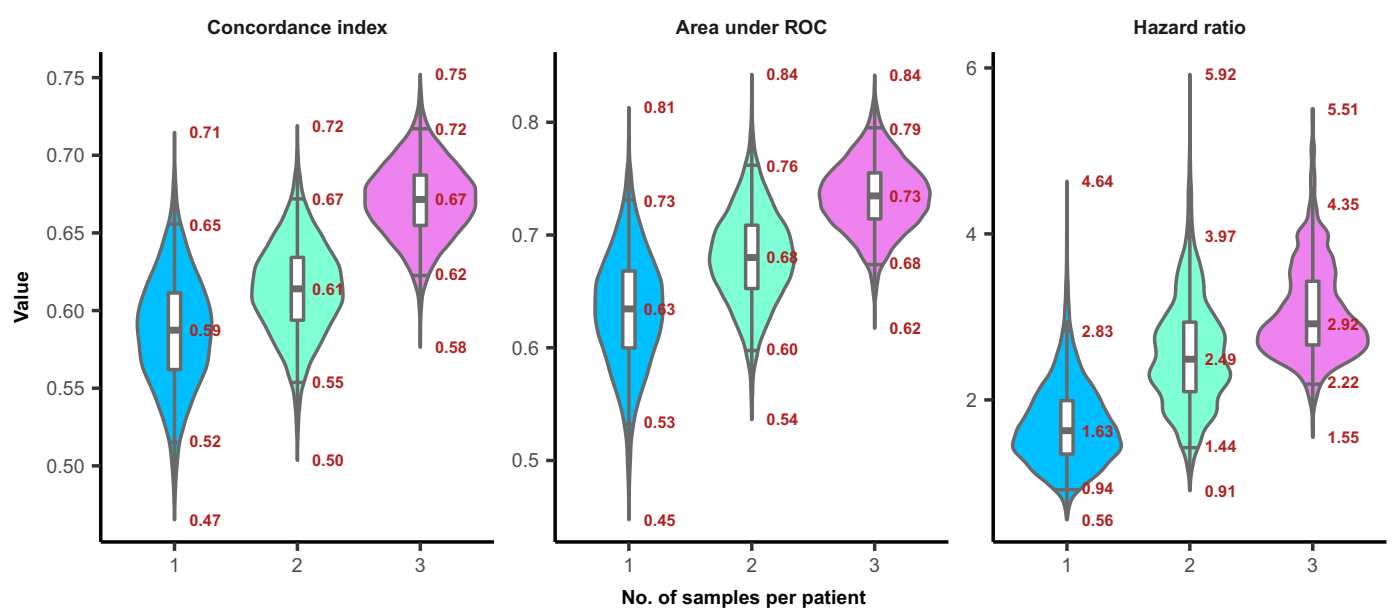

Figure 4. Violin plots depicting distributions of the major indicators of the signature performance, including concordance index (CI), area under ROC (AUC) and hazard ratio (HR), obtained using one, two and three randomly chosen samples per patient to assess patient risk. Annotations show minimum, $2.5^{\text {th }}$ percentile, median, $97.5^{\text {th }}$ percentile and maximum of the given distributions.

Considering CI, HR and AUC and a full set of patient samples we have validated results obtained by the original study ${ }^{9}$. Current results demonstrate the robust predictive performance of the 4-gene signature, and thus verify our previous results using an independent patient cohort and a new gene expression assay platform. In addition, we showed that the prediction accuracy depends on the number of separate margin samples across which the expression data are integrated.

Since genetic aberrations may spread from the tumor asymmetrically, they may be undetectable in some of the obtained margins ${ }^{9}$. A greater number of available margin samples increases the chance of detection of these aberrations and enables a more precise quantification of patient risk. Therefore, the inclusion of multiple histologically negative margin samples per patient for gene expression analysis is one of the strengths of our study. Our results show that the current signature demonstrates sufficient prediction accuracy if at least three separate margins per patient are used. This requirement is clinically feasible and should not prevent utilization of the signature in clinical practice.

Pathway enrichment analysis using the signature genes and their PPI partners revealed their involvement in several pathways known to be associated with OSCC development. These include organization of the extracellular matrix, integrin interactions and collagen synthesis; changes of which are essential for many malignant processes in OSCC ${ }^{12}$. Similarly, PI3K-Akt signalling alterations are observed in multiple cancers, and are also believed to be associated with cancer drug resistance ${ }^{13}$. A recently identified partial-epithelial to mesenchymal transition (p-EMT) program has been shown as an independent predictor of nodal metastasis, tumor grade and additional pathological features indicating aggressive tumor behaviour in oral carcinoma ${ }^{14}$. Notably, our signature genes are among the study findings, with MMP1 being over-expressed in p-EMT cells, and COL4A1, P4HA2 and THBS2 up-regulated upon TGFb treatment, which induced a p-EMT-like program and increased invasive potential, in the SCC9 oral cancer cell line. This study corroborates our findings, and we highlight the existence of molecular alterations in surrounding tumor tissues, including surgical resection margins, as drivers of disease recurrence.

Our findings have translational relevance for the management of patients with OSCC. Patients with a diagnosis of histologically negative margins are often considered to have a lower risk of recurrence; however, we demonstrate that a subset of these patients develops recurrent disease and that recurrence can be predicted by gene expression changes. In the absence of other clinical indicators, prediction of a high risk of local recurrence in patients with histologically negative margins could thus be used to modify post-operative treatment options and surveillance. We suggest clinical utilization of this 4-gene signature for molecular analysis of histologically negative margins from patients undergoing OSCC surgical resection as part of post resection clinical management.

\section{Material and Methods}

This is a retrospective, single center cohort study aimed to independently validate the 4-gene prognostic signature. This study complies with the Guidelines for the REporting of tumor MARKer Studies (REMARK) ${ }^{15}$, and followed the guidelines outlined in the Transparent Reporting of a multivariant prediction model for Individual Prognosis Or Diagnosis (TRIPOD) statement ${ }^{16}$. Original, raw data were submitted to Gene Expression Omnibus (GEO), and are publicly available under GEO series number GSE108712.

Ethics declaration - study participants. This work was performed under approval of the University Health Network Research Ethics Board (REB approval number 02-0488-C). The study followed the ethics recommendations from the Helsinki Declaration. Informed consent was obtained from all patients before sample collection. Patients included in the study were diagnosed and treated at the Princess Margaret Cancer Centre/ Toronto General Hospital, Toronto, ON, Canada. Patient inclusion criteria were: (i) primary, surgically resected OSCC, (ii) patients having a final pathology report confirming that all surgical resection margins were histologically normal or negative. 
Clinical specimens and study design. Sample size. We retrospectively collected formalin-fixed, paraffin embedded (FFPE) tissue blocks from 277 margin samples from 67 patients. Of these, 8 samples were removed since they were technical replicates. Additional 24 samples from five patients with insufficient follow up time (follow up $<3$ years). This study was conducted on 245 margins samples obtained from a total of 62 patients, each with more than 3 years of follow-up. We have analyzed up to 8 surgical resection margins per patient, with an average of 4 margins per patient. All margins were histologically normal (i.e., negative), as determined by final histopathological analysis (BP-O). None of the patients received chemo or radiation therapy before surgery and sample collection.

Source of data. Sample accrual and retrieval was performed starting in March, 2011 and ending in March, 2012. RNA was extracted in April-May, 2012. Gene expression analysis was performed in June, 2012. Samples were randomized and their identities anonymized using the Random Sampling feature in Microsoft Excel prior to gene expression analysis.

Outcome measures. Patients were classified into two groups: (1) patients experiencing local recurrence and (2) recurrence-free patients. Recurrence status was updated in September, 2019 (C.S., clinical research assistant). Pathology reports were examined to confirm recurrence status, which is established by the head and neck oncology team based on standard examination procedures for patients with OSCC (PG, JI).

Procedures. FFPE tissues from histologically normal surgical resection margins were cut ( $4-5 \mu \mathrm{m}$ tick), stained with Hematoxylin-Eosin and subjected to needle microdissection using the stereo microscope Leica EZ4 (Leica Microsystems, Wetzlar, Germany) before RNA extraction, in order to isolate pure normal cell populations from margin tissues. RNA from FFPE samples was isolated using the RecoverAll Total Nucleic Acid Isolation kit (Ambion/Life Technologies, Carlsbad, CA, USA), following our previously reported protocol with modifications to improve RNA yield ${ }^{17}$. RNA was stored at $-80^{\circ} \mathrm{C}$ until RNA extraction and gene expression validation. In addition, tumor tissues from the same patients were collected to confirm whether the 4-gene signature was also deregulated in tumors, in the same direction (up-regulation) as compared to surgical resection margins.

Two Nanostring nCounter ${ }^{\mathrm{TM}}$ probes were designed against each gene of the 4-gene signature (MMP1, COL4A1, P4HA2 and THBS2), being comprised of one capture probe linked to biotin and one reporter probe attached to a color-coded molecular tag for each gene sequence, according to the nCounter ${ }^{\mathrm{TM}}$ code-set design. RNA samples randomized by a numerical ID were subjected to NanoString nCounter ${ }^{\mathrm{TM}}$ analysis by the Princess Margaret Genomics Centre (https://www.pmgenomics.ca) facility, Toronto, ON, Canada.

Patient samples were analyzed in two batches consisting of 125 and 152 margins tissue samples. The detailed protocol for mRNA transcript quantification analysis, including sample preparation, hybridization, detection and scanning followed the manufacturer's recommendations and was described in ${ }^{18}$. We used $400 \mathrm{ng}$ of total RNA isolated from FFPE tissues for detection of probe signals. Raw data were analyzed using the nCounter ${ }^{\mathrm{TM}}$ digital analyzer software (http://www.nanostring.com/support/ncounter), and gene expression levels were subjected to further bioinformatic and statistical analyses, as described below.

Missing data. Clinicopathological characteristics of the patients comprised several missing values. Therefore, statistics reported in the Table 1, assessing prognostic properties of the individual characteristics, were calculated across the subset of patients without missing values. Number of patients used to calculate these statistics is indicated in the table $(\mathrm{N})$. As there were no missing signature genes expression values, no data imputation was applied.

Statistical analysis. Gene expression data were normalized and background corrected, using R package NanoStringNorm v1.1.21 ${ }^{19}$. Expressions were then normalized for technical assay variation using positive controls and background-corrected using negative controls. Subsequently, data were normalized for sample/ RNA content variation by applying the housekeeping genes normalization method (housekeeping.geo.mean), as described in NanoString nCounter ${ }^{\circledR}$ Expression Data Analysis Guide. Two housekeeping genes, GAPDH and RPS18 were used as internal controls, as these have shown stable expression levels in our previous studies using normal and tumor tissues from head and neck cancer patients ${ }^{9,18,20,21}$. Normalized expressions levels were log-transformed and subsequently converted to Z-scores. Data were submitted to Gene Expression Omnibus (GEO), and are publicly available under GEO series number GSE108712.

Proportional risks were calculated as a linear combination of the Z-score-transformed expressions of $M M P 1$, COL4A1, P4HA2 and THBS2, applying original signature coefficients $(0.25,0.63,0.45$ and 0.34 , respectively) as described by Reis et al. ${ }^{9}$. Patients were then stratified into two groups according to their proportional risks using the chosen threshold (see Results section). Hazard ratio between the two patient groups and associated p-values were then calculated using the survdiff function from the R package survival (version 2.38.3) ${ }^{22}$. Kaplan-Meier plots were generated using function survfit from the same package. Receiver operating characteristics (ROC) curve, area under ROC curve (AUC), and prediction accuracy were obtained using functions provide by the $\mathrm{R}$ package AUC (version 0.3.0 $)^{23}$.

Risk groups. When assessing the signature, patients were stratified into two groups according to predicted proportional risks, first using its median as a threshold, then using the optimized threshold that was selected to maximize significance of the resulting hazard ratio.

Development vs. validation data. The current validation was conducted using data from a fully independent cohort of patients, with no overlap with the cohort used for the signature development, or its first previously reported validation study ${ }^{9}$. Differences between the clinical and histopathological characteristics of the development and validation cohorts are summarized in Table 1. 
Identification of protein-protein interaction partners and comprehensive pathway enrichment analysis. Physical protein interaction (PPI) partners of the 4 signature genes were obtained using the Integrated Interaction Database v2016-10 (IID; http://ophid.utoronto.ca/iid) ${ }^{24}$. We considered only interactions that were experimentally validated or computationally predicted in head and neck tissue. Subsequently, we performed a comprehensive pathway enrichment analysis using Pathway Data Integration Portal v2.5.9.3 (pathDIP; http://ophid. utoronto.ca/pathDIP $)^{25}$.

Received: 11 December 2019; Accepted: 17 January 2020;

Published online: 03 February 2020

\section{References}

1. Petersen, P. E. Oral cancer prevention and control - The approach of the World Health Organization. Oral Oncol. 45, 454-460 (2009).

2. Bray, F. et al. Global cancer statistics 2018: GLOBOCAN estimates of incidence and mortality worldwide for 36 cancers in 185 countries. CA. Cancer J. Clin. 68, 394-424 (2018).

3. Anderson, C. R., Sisson, K. \& Moncrieff, M. A meta-analysis of margin size and local recurrence in oral squamous cell carcinoma. Oral Oncol. 51, 464-469 (2015).

4. Feller, L. \& Lemmer, J. Oral Squamous Cell Carcinoma: Epidemiology, Clinical Presentation and Treatment. J. Cancer Ther. 03, 263-268 (2012).

5. Weijers, M., Snow, G. B., Bezemer, P. D. \& Van Der Waal, I. The clinical relevance of epithelial dysplasia in the surgical margins of tongue and floor of mouth squamous cell carcinoma with regard to local recurrence; an analysis of 37 patients. J Oral Pathol Med. 37, 11-15 (2002).

6. Leemans, C. R., Tiwari, R., Nauta, J. J. P., Waal, I. V. D. \& Snow, G. B. Recurrence at the primary site in head and neck cancer and the significance of neck lymph node metastases as a prognostic factor. Cancer 73, 187-190 (1994).

7. Nathan, C. A. O. et al. Molecular analysis of surgical margins in head and neck squamous cell carcinoma patients. Laryngoscope 112, 2129-2140 (2002).

8. Bilde, A., Von Buchwald, C., Dabelsteen, E., Therkildsen, M. H. \& Dabelsteen, S. Molecular markers in the surgical margin of oral carcinomas. J. Oral Pathol. Med. 38, 72-78 (2009).

9. Reis, P. P. et al. A gene signature in histologically normal surgical margins is predictive of oral carcinoma recurrence. BMC Cancer 11, 437 (2011).

10. Geiss, G. K. et al. Direct multiplexed measurement of gene expression with color-coded probe pairs. Nat. Biotechnol. 26, 317-325 (2008).

11. Moons, K. G. M., Altman, D. G., Vergouwe, Y. \& Royston, P. Prognosis and prognostic research: Application and impact of prognostic models in clinical practice. BMJ 338, 1487-1490 (2009).

12. Ziober, A. F., Falls, E. M. \& Ziober, B. L. The extracellular matrix in oral squamous cell carcinoma: friend or foe? Head Neck 28, $740-9(2006)$

13. Vara, J. Á. F. et al. P13K/Akt signalling pathway and cancer. Cancer Treat. Rev. 30, 193-204 (2004)

14. Puram, S. V. et al. Single-cell transcriptomic analysis of primary and metastatic tumor ecosystems in head and neck cancer. Cell 171, 1611-1624 (2017).

15. McShane, L. M. et al. REporting recommendations for tumor MARKer prognostic studies (REMARK). Br. J. Cancer 39, 387-91 (2005).

16. Collins, G. S., Reitsma, J. B., Altman, D. G. \& Moons, K. G. M. Transparent reporting of a multivariable prediction model for individual prognosis or diagnosis (TRIPOD): the TRIPOD statement. Ann Intern Med. 6, W1-73 (2015).

17. Goswami, R. S. et al. MicroRNA signature obtained from the comparison of aggressive with indolent non-hodgkin lymphomas: Potential prognostic value in mantle-cell lymphoma. J. Clin. Oncol. 31, 2903-2911 (2013).

18. Reis, P. P. et al. MRNA transcript quantification in archival samples using multiplexed, color-coded probes. BMC Biotechnol. 11, 46 (2011).

19. Waggott, D. et al. NanoStringNorm: An extensible R package for the pre-processing of nanostring mRNA and miRNA data. Bioinformatics 28, 1546-1548 (2012).

20. Bertonha, F. B. et al. PHF21B as a candidate tumor suppressor gene in head and neck squamous cell carcinomas. Mol. Oncol. 9 , 450-462 (2015).

21. Reis, P. P. et al. Programmed cell death 4 loss increases tumor cell invasion and is regulated by miR-21 in oral squamous cell carcinoma. Mol. Cancer 9, 238-250 (2010).

22. Ballings, M. \& Van den Poel, D. Threshold Independent Performance Measures for Probabilistic Classification Algorithms (2013).

23. Therneau, T. M. \& Grambsch, P. M. Modeling Survival Data: Extending the Cox Model. Statistics for Biology and Health. (Springer, 2000).

24. Kotlyar, M., Pastrello, C., Sheahan, N. \& Jurisica, I. Integrated interactions database: Tissue-specific view of the human and model organism interactomes. Nucleic Acids Res. 44, D536-D541 (2016).

25. Rahmati, S., Abovsky, M., Pastrello, C. \& Jurisica, I. PathDIP: An annotated resource for known and predicted human gene-pathway associations and pathway enrichment analysis. Nucleic Acids Res. 45, D419-D426 (2017).

\section{Acknowledgements}

This study was supported by the Ontario Institute for Cancer Research (SKR) and The Galloway Research Fund/ PMH Foundation (SKR, RG). I.J. was supported in part by funding from Natural Sciences Research Council (NSERC \#203475), Canada Foundation for Innovation (CFI \#225404, \#30865), Canada Research Chair Program (CRC \#203373, \#225404), Ontario Research Fund (RDI \#34876), Ontario Research Fund (GL2-01-030), IBM and Ian Lawson van Toch Fund. The funders had no role in study design, data collection and analysis, decision to publish, or preparation of the manuscript.

\section{Author contributions}

P.P.R., S.K.R. and I.J. designed the study. P.P.R., R.S.G., Y.X. and M.S. collected the data. P.P.R., R.S.G., A.L.S., L.E.S.M., T.T. and I.J. analyzed and interpreted the data. T.T. and I.J. performed bioinformatics and statistical analysis. B.P.-O., C.S., D.G., D.B., R.G., P.G. and J.I. collected, reviewed and updated clinical and histopathological data and patient follow-up. P.P.R., T.T., R.G., L.E.S.M., M.S., S.K.R. and I.J. wrote the manuscript. All authors reviewed the manuscript and approved the final version and are accountable for all aspects of this work. 


\section{Competing interests}

The authors declare no competing interests.

\section{Additional information}

Supplementary information is available for this paper at https://doi.org/10.1038/s41598-020-58688-y.

Correspondence and requests for materials should be addressed to P.P.R., I.J. or S.K.-R.

Reprints and permissions information is available at www.nature.com/reprints.

Publisher's note Springer Nature remains neutral with regard to jurisdictional claims in published maps and institutional affiliations.

(c) (1) Open Access This article is licensed under a Creative Commons Attribution 4.0 International License, which permits use, sharing, adaptation, distribution and reproduction in any medium or format, as long as you give appropriate credit to the original author(s) and the source, provide a link to the Creative Commons license, and indicate if changes were made. The images or other third party material in this article are included in the article's Creative Commons license, unless indicated otherwise in a credit line to the material. If material is not included in the article's Creative Commons license and your intended use is not permitted by statutory regulation or exceeds the permitted use, you will need to obtain permission directly from the copyright holder. To view a copy of this license, visit http://creativecommons.org/licenses/by/4.0/.

(C) The Author(s) 2020 